\title{
Comparison of the Self Diffusion Coefficient of Polystyrene in Solution Estimated by Forced Rayleigh Scattering and Fluorescence Recovery after Photobleaching
}

\author{
Tadashi InOUE, Norio Nemoto, ${ }^{*}$ Takaharu KoJima, \\ and Michio KURATA \\ Institute for Chemical Research, Kyoto University, \\ Uji, Kyoto 611, Japan
}

(Received May 11, 1988)

\begin{abstract}
The self diffusion coefficient $D_{\mathrm{s}}$ of two labeled polystyrene (PS) samples in dibutyl phthalate (DBP) has been measured in the range of polymer concentration from 0.1 to $50 \mathrm{wt} \%$ using forced Rayleigh scattering (FRS) and fluorescence recovery after photobleaching (FRAP). 2-Nitro-4-carboxy-4'-dimethylaminostilbene and 4-glycyl-7-nitrobenzofurazan have been used as photochromic dyes for FRS and FRAP measurements, respectively. Good agreement has been found between two sets of $D_{\mathrm{s}}$ data in the range of $D_{\mathrm{s}}$ from $10^{-8}$ to $10^{-12} \mathrm{~cm}^{2} \mathrm{~s}^{-1}$ within experimental error. In the dilute region of $c \leq 1 \mathrm{wt} \%$, mutual diffusion coefficient $D$ of unlabeled but otherwise the same PSs in DBP has been measured by dynamic light scattering. The $D_{\mathrm{s}}$ assymptotically approached $D$ with decreasing polymer concentration.
\end{abstract}

KEY WORDS Self Diffusion Coefficient / Forced Rayleigh Scattering /

Fluorescence Recovery after Photobleaching / Polystyrene Solution /

Photobleachable Dye /

The self diffusion coefficient $D_{\mathrm{s}}$ is a most useful quantity for the study of slow chain dynamics in condensed polymeric systems. ${ }^{1}$ Among various techniques proposed for $D_{\mathrm{s}}$ measurements, ${ }^{2-13}$ both forced Rayleigh scattering (FRS) and fluorescence recovery after photobleaching (FRAP) are methods which make use of photochromic dyes for labeling of polymers. The former method has been developed in the field of physical chemistry of synthetic polymers, while the latter has been invented in the field of biophysics. ${ }^{14-17}$ Both methods have merits and demerits. The FRS method usually provides an accurate estimate of $D_{\mathrm{s}}$ because of high diffracted intensity $I_{\mathrm{s}}$ detected at the Bragg angle and of a definite value of $I_{\mathrm{s}}$ at time $t \rightarrow \infty$, i.e., the base line value. However, the complicated photochemical process of a dye sometimes gives rise to an anomalous $I_{\mathrm{s}} v s$. $t$ curve, which hinders unambiguous data analysis. ${ }^{18,21}$ The FRAP method is free from the dye problem, but an indefinite value of $I_{\mathrm{s}}$ at $t \rightarrow \infty$ makes it difficult to estimate $D_{\mathrm{s}}$ accurately. ${ }^{22}$ Furthermore it is troublesome in a strict sense that there is no standard material with a known $D_{\mathrm{s}}$ value with which accuracy of the either measurement can be examined. On the other hand, it is reasonable to consider that the two techniques should give an identical $D_{\mathrm{s}}$ value in principle, when applied to the same polymer-solvent system. Then good agreement for the absolute magnitude of $D_{\mathrm{s}}$ may be obtained. In spite of the importance of the two techniques, such com-

\footnotetext{
* To whom all correspondence should be addressed.
} 
parison has not been attempted yet.

Recently we constructed an apparatus with which both FRS and FRAP measurements can be performed with a slightly different experimental set up. ${ }^{23}$ In this paper, we compare two sets of $D_{\mathrm{s}}$ data of narrow-distribution polystyrene in dibutyl phthalate over a wide range of concentration from 1 to $50 \mathrm{wt} \%$ obtained with the FRS and the FRAP methods. Dynamic light scattering measurements have been also made on unlabeled but otherwise the same polymer solutions in the dilute region of $c \leq 1 \mathrm{wt} \%$, and the mutual diffusion coefficient $D$ data obtained have been compared with corresponding $D_{\mathrm{s}}$ data from FRS measurements. This experiment was aimed to confirm that, in the very dilute region, $D_{\mathrm{s}}$ should assymptotically approach $D$ with decreasing polymer concentration.

\section{EXPERIMENTAL}

\section{Materials}

Two narrow distribution polystyrenes $\left(\bar{M}_{w}=\right.$ 43900; sample code F4 and $\bar{M}_{w}=355000$; code F40, Toyo Soda) were used as samples in this study. The polymers were randomly chloromethylated in methylene dichloride with chloromethylmethylether and with zinc chloride as catalyst. The chloromethylated polymers were subsequently labeled with a photobleachable dye of 2-nitro-4-carboxy-4'dimethylaminostilbene for FRS measurements and with a photobleachable fluorescent dye of 4-glycyl-7-nitrobenzofurazan for FRAP measurements, respectively. The detailed labeling procedure is described elsewhere. ${ }^{24}$ GPC combined with low-angle light scattering measurements has shown that there is no change in either molecular weight or molecular weight distribution of the polymers during the labeling procedure.

Distilled dibutyl phthalate (DBP) was used as solvent for preparation of solutions to be measured. Weighed amounts of labeled PS and DBP were dissolved in sufficient amount of
$\mathrm{CH}_{2} \mathrm{Cl}_{2}$, stirred for a couple of days to complete homogeneous mixing, and then filtered through a $0.45 \mu \mathrm{m}$ pore size filter. Methylenedichloride was subsequently evaporated very slowly from the solution with occasional stirring to obtain a test polymer solution. The polymer concentration $c$ was determined by weighing. The range of $c$ investigated was from 0.1 to $50 \mathrm{wt} \%$.

\section{Methods}

The self diffusion coefficient $D_{\mathrm{s}}$ of labeled PS in solutions was measured using a computercontrolled apparatus recently constructed at our laboratory. ${ }^{23}$ For the FRS experiment, monochromatic light of an argon-ion laser $(\hat{\lambda}=488 \mathrm{~nm})$ and a He-Ne laser $(\lambda=633 \mathrm{~nm})$ were used as the writing and the reading beams, respectively. The optical configuration of the apparatus is similar to those of FRS instruments reported in the literature..$^{2,4,8}$ Acquisition and analysis of diffracted light intensity data were conducted with a homemade data processor. The sample was sandwiched between two $1.5 \mathrm{~cm}$ diameter fusedsilica optical windows, or put into a $1 \mathrm{~cm}$ square-shaped cell with width of $1-3 \mathrm{~mm}$, which was fixed in the cell holder. The holder was thermoregulated within $\pm 0.02^{\circ} \mathrm{C}$ by circulating a mixture of ethyleneglycol and water through an outer bath and an external thermostating bath.

For the FRAP experiment, a sample was deposited as a thin film on pattern mask (pitch 4-64 $\mu \mathrm{m}, 1 \mathrm{~mm}$-square) made by electron-ray lithography on a $1.5 \mathrm{~cm}$ diameter fused-silica optical window. A laser line $(\lambda=488 \mathrm{~nm})$ with an output of $300 \mathrm{~mW}$ was used for photobleaching of the labeled PS at time $t=0$ and also for fluorescence recovery measurements at a later time by decreasing the intensity by a factor of $10^{5}$. Since continuous illumination was found to photobleach the fluorescent dye even with such a low intensity, the samples were illuminated only for short intervals in detecting fluorescence signals from the samples 
by use of an clock-controlled rotating shutter. All experiments were performed at $25 \pm$ $0.02^{\circ} \mathrm{C}$.

Dynamic light scattering measurements on unlabeled but otherwise the same PS-DBP solutions were made at $25 \pm 0.01^{\circ} \mathrm{C}$ with the instrument reported earlier. ${ }^{25}$

\section{RESULTS AND DISCUSSION}

\section{Data Analysis}

Figure 1 shows the time profiles of diffracted light intensity $I_{\mathrm{d}}$ and fluorescence intensity $I_{\mathrm{f}}$ as an example of raw data obtained for a $5 \mathrm{wt} \%$ solution of PS with $\bar{M}_{w}=355000$ in DBP with FRS and FRAP, respectively. The solid curve in Figure 1(a) is a curve fitted with eq 1 , the theoretical model function with a single decay rate $\Gamma_{\mathrm{d}}$ for FRS.

$$
I_{\mathrm{d}}=\left\{A \exp \left(-\Gamma_{\mathrm{d}} t\right)+B\right\}^{2}+C^{2}
$$

Here $A$ is amplitude, $B$ and $C$ are contributions from coherent and incoherent background optical field, respectively. It is evident from the figure that eq 1 expresses the time dependence of $I_{\mathrm{d}}$ quite well. Indeed, normal residuals from the fit are very small and random as shown in the figure. Coherent scattered intensity from the polymer solutions was found to be negligibly small in comparison with the diffracted light intensity at $t=0$, i.e., a value of $A$, so that fitting by eq 1 with $B=0$




(a)



(b)

Figure 1. (a) Decay of diffracted light intensity $I_{\mathrm{d}}$ due to self-diffusion of labeled PS with $\bar{M}_{w}=355000$ in DBP. Polymer concentration is $5 \mathrm{wt} \%$ and the grating spacing $d$ is $8 \mu \mathrm{m}$. The solid curve is a least-square fit with eq 1. (b) Recovery of fluorescence intensity $I_{\mathrm{f}}$ due to self diffusion of labeled PS with $\bar{M}_{w}=355000$ in DBP. Polymer concentration is $5 \mathrm{wt} \%$ and the pitch of the striped pattern $d$ is $8 \mu \mathrm{m}$. The solid curve is a least-square fit with eq 2 . Normal residuals obtained from each fit are shown in the bottom of the each figure. 
gave only a few percent different value to $\Gamma_{\mathrm{d}}$. Figure 1(b) shows a recovery profile of fluorescence intensity $I_{\mathrm{f}}$. Data are more scattered in comparison with the FRS data shown in Figure 1(a) because of low irradiation intensity of the laser beam used for detection of $I_{\mathrm{f}}$. The data were fitted with eq 2 in which higher frequency terms were neglected.

$$
I_{\mathrm{f}}=A^{\prime}-B^{\prime} \exp \left(-\Gamma_{\mathrm{f}} t\right)
$$

Here $A^{\prime}$ is the value of $I_{\mathrm{f}}$ in equilibrium and $B^{\prime}$ is the amount of $I_{\mathrm{f}}$ which is recovered due to self diffusion of labeled polymers. Normal residuals from the fit are shown in the bottom of the figure. Fitting by the solid curve in the figure gave $\Gamma_{\mathrm{f}}$ to an accuracy of about $30 \%$. Inclusion of higher frequency terms did not improve the fitting. No PS-DBP solutions tested by either FRS or FRAP methods gave an anomalous $I$ vs. $t$ curve with a minimum and a maximum, and the curves could be successfully fitted with the corresponding eq 1 or 2.

Figure 2 shows that $\Gamma_{\mathrm{d}}$ and $\Gamma_{\mathrm{f}}$ thus obtained satisfy the proportionality of the decay rate to the square of the wave vector $q(=2 \pi / d, d$, the pitch of the interference (FRS) or the striped pattern (FRAP)). It may be noted that $q^{2}$ was widely changed by a factor of 64 for FRAP measurements. $D_{\mathrm{s}}$ was estimated from eq 3 to an accuracy of 10 and $30 \%$ for FRS and FRAP, respectively.

$$
D_{\mathrm{s}}=\Gamma_{i} / q^{2} \quad(i=d, f)
$$

Values of $D_{\mathrm{s}}$ are given in Table I.

Normalized time correlation function data of scattered intensity from unlabeled PS solutions measured by dynamic light scattering (DLS) were analyzed using a single exponential type of decay function. The mutual diffusion coefficient $D$ was estimated as the slope of the decay rate $\Gamma v s . q^{2}$ plot within an error of a few percent. Values of $D$ are given in Table I.

$D_{\text {s }}$ obtained by FRS and FRAP and also $D$ by DLS are plotted against polymer con-



(a)

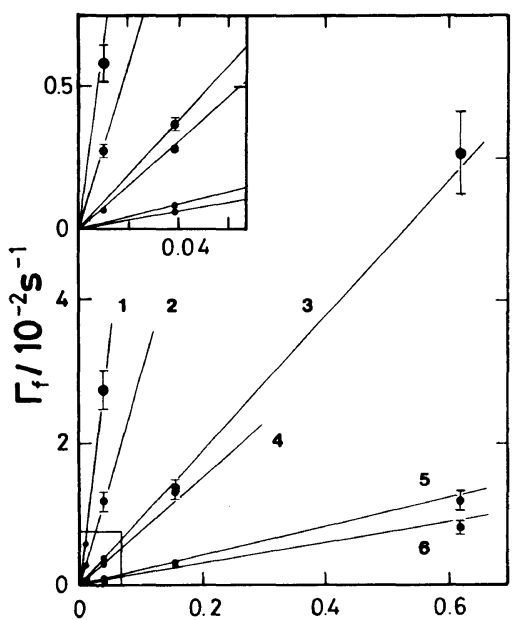

(b)

$q^{2} / \mu m^{-2}$

Figure 2. Plots of relaxation rate $\Gamma_{i}(i=d, f)$ against the square of the wave vector $q . D_{\mathrm{s}}$ is estimated as a slope of a straight line drawn in the figure for each $c$ and $M$. (a) $\Gamma_{\mathrm{d}}$ from FRS. Sample and concentration are: 1, F40, $10 \mathrm{wt} \%$; 2, F4, 30\%; 3, F40, $15 \%$; 4, F4, $40 \%$. (b) $\Gamma_{\mathrm{f}}$ from FRAP. Sample and concentration are: $1, \mathrm{~F} 40,1 \mathrm{wt} \% ; 2$, $\mathrm{F} 40,2 \%$; 3, F40, 5\%; 4, F4, 20\%; 5, F40, $10 \%$; and 6, F4, $30 \%$.

centration $c$ in Figure 3 for comparison. In the dilute regime where $c$ is less than $1 \mathrm{wt} \%, D$ of the both samples looks almost independent of $c$ in the logarithmic scale, and a value at infinite dilution, i.e., the translational diffusion coefficient $D_{0}$, could be easily estimated as $2.83 \pm 0.04 \times 10^{-8}$ and $9.30 \pm 0.10 \times 10^{-9}$ 
Table I. $D_{\mathrm{s}}$ values of PS with $\bar{M}_{\mathrm{w}}=43900(\mathrm{~F} 4)$ and 355000 (F40) in DBP estimated by FRS and

FRAP, and $D$ values of unlabeled but otherwise the same PSs in DBP by DLS at $25^{\circ} \mathrm{C}$

\begin{tabular}{|c|c|c|c|c|}
\hline \multirow{2}{*}{$\bar{M}_{w} \times 10^{-4}$} & $c / \mathbf{w t} \%$ & $D_{\mathrm{s}} / \mathrm{cm}^{2} \mathrm{~s}^{-1}$ & $c / \mathrm{wt} \%$ & $D_{\mathrm{s}} / \mathrm{cm}^{2} \mathrm{~s}^{-1}$ \\
\hline & \multicolumn{2}{|c|}{ by FRS } & \multicolumn{2}{|c|}{ by FRAP } \\
\hline \multirow[t]{9}{*}{4.39} & 0.100 & $2.5 \times 10^{-8}$ & & \\
\hline & 0.300 & $2.5 \times 10^{-8}$ & & \\
\hline & 0.995 & $2.3 \times 10^{-8}$ & 0.996 & $2.2 \times 10^{-8}$ \\
\hline & 10.1 & $6.5 \times 10^{-9}$ & 9.67 & $5.3 \times 10^{-9}$ \\
\hline & 19.9 & $9.6 \times 10^{-10}$ & 19.8 & $7.7 \times 10^{-10}$ \\
\hline & 30.4 & $1.5 \times 10^{-10}$ & 29.8 & $1.5 \times 10^{-10}$ \\
\hline & 39.6 & $2.6 \times 10^{-11}$ & 38.5 & $2.5 \times 10^{-11}$ \\
\hline & 40.7 & $1.7 \times 10^{-11}$ & 40.8 & $1.9 \times 10^{-11}$ \\
\hline & 49.9 & $1.7 \times 10^{-12}$ & 49.7 & $1.4 \times 10^{-12}$ \\
\hline \multirow[t]{7}{*}{35.5} & 0.098 & $1.0 \times 10^{-8}$ & & \\
\hline & 0.300 & $8.5 \times 10^{-9}$ & & \\
\hline & 1.06 & $8.3 \times 10^{-9}$ & 1.03 & $7.1 \times 10^{-9}$ \\
\hline & 2.05 & $4.0 \times 10^{-9}$ & 2.01 & $3.0 \times 10^{-9}$ \\
\hline & 5.38 & $1.1 \times 10^{-9}$ & 4.74 & $9.6 \times 10^{-10}$ \\
\hline & 10.5 & $1.9 \times 10^{-10}$ & 10.1 & $2.1 \times 10^{-10}$ \\
\hline & 20.0 & $1.3 \times 10^{-11}$ & 20.4 & $1.3 \times 10^{-11}$ \\
\hline
\end{tabular}

\begin{tabular}{cll}
\hline \multirow{2}{*}{$\bar{M}_{w} \times 10^{-4} \frac{c / \mathrm{wt} \%}{D_{\mathrm{s}} / \mathrm{cm}^{2} \mathrm{~s}^{-1}}$} & \multicolumn{1}{c}{ by DLS } \\
\hline \multirow{2}{*}{4.39} & 0.100 & $2.79 \times 10^{-8}$ \\
& 0.300 & $2.91 \times 10^{-8}$ \\
35.5 & 1.00 & $2.77 \times 10^{-8}$ \\
& 0.100 & $9.41 \times 10^{-9}$ \\
& 0.296 & $9.87 \times 10^{-9}$ \\
& 1.00 & $1.11 \times 10^{-8}$ \\
\hline
\end{tabular}

$\mathrm{cm}^{2} \mathrm{~s}^{-1}$ to PS molecule with $\bar{M}_{w}=43900$ (F4) and 355000 (F40), respectively. $D_{\mathrm{s}}$ is almost equal to or slightly less than $D$ in this concentration range as is expected to a polymergood solvent system, and tends to approach assymptotically $D_{0}$ with decreasing concentration. Since DLS is a well-established technique for an accurate estimate of $D_{0}$, good agreement between $D_{\mathrm{s}}$ and $D_{0}$ at infinite dilution indicates that $D_{\mathrm{s}}$ can be estimated to high accuracy with our instrument. The hydrodynamic radius $R_{\mathrm{H}}$ of the polymer in DBP can be calculated from values of $D_{0}$ and solvent viscosity by applying the Einstein-Stokes re-

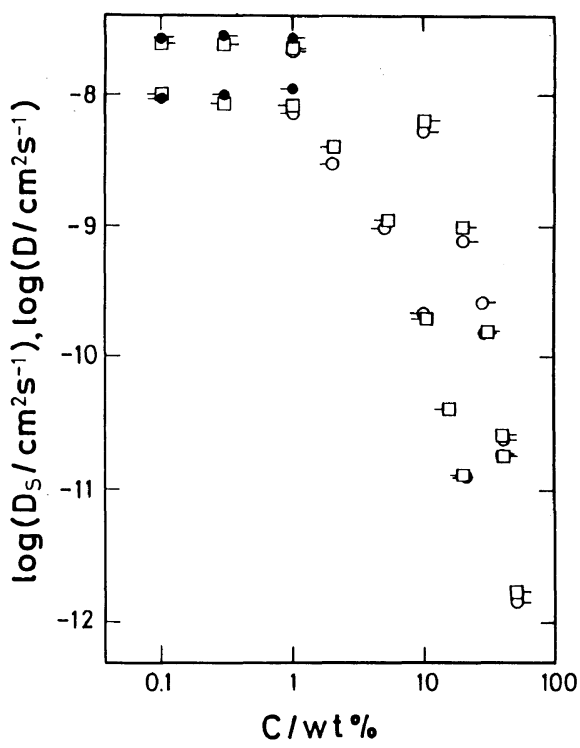

Figure 3. Comparison of $D_{\mathrm{s}}$ estimated by FRS and FRAP, and $D$ by DLS. Symbols: ( $\square$ ), FRS data; $(\bigcirc)$, FRAP data; and (O), DLS data. The right pip denotes F4 sample $\left(\bar{M}_{w}=43900\right)$ and the left F40 sample $\left(\bar{M}_{w}=\right.$ 355000).

lationship. It has been found that the dependence of $R_{\mathrm{H}}$ on molecular weight is closely represented by eq 4 , which was determined for PS in good solvent in an earlier study. ${ }^{26}$

$$
R_{\mathrm{H}}=1.60 \times 10^{-9} \bar{M}_{w}{ }^{0.55}
$$

In the region of $c \geq 1 \mathrm{wt} \%$, two sets of $D_{\mathrm{s}}$ data for each PS sample are now compared. As a general trend, $D_{\mathrm{s}}$ values obtained by FRAP look a little smaller than corresponding values by FRS. Taking into account the experimental uncertainties involved in $D_{\mathrm{s}}$ values by FRAP, however, we may state that they are located on a single composite curve for each molecular weight. Thus we may conclude that correct values of $D_{\mathrm{s}}$ from $10^{-8}$ to $10^{-12} \mathrm{~cm}^{2} \mathrm{~s}^{-1}$ can be obtained for the PS-DBP system with either FRS or FRAP.

The concenteration dependence of $D_{\mathrm{s}}$ can not be described by a simple power law as is clear from Figure 3. Steep decrease in $D_{\mathrm{s}}$ of the low molecular weight PS at high concentration above $30 \mathrm{wt} \%$ is mainly due to increase in local 
friction or decrease in free volume which essentially determines the mobility of a polymer segment in condensed system. On the other hand, the concentration dependence of $D_{\mathrm{s}}$ of the higher molecular weight PS should be interpreted as being due to the combined effects of local friction, hydrodynamic interaction and topological interaction. ${ }^{27} \mathrm{De}$ pendence of $D_{\mathrm{s}}$ on temperature, concentration and molecular weight in the concentrated regime will be discussed in detail in future papers.

Acknowledgments. We are very grateful to Prof. H. Sillescu of Mainz University for sending us a prescription for the synthesis of the photobleachable dye. We also thank to Prof. S. Tanimoto, Drs. A. Toshimitsu and T. Oida of this institute for their help and advice in labeling polystyrene. Thanks are also due to Prof. H. Yu of University of Wisconsin for his helpful criticism and discussion.

\section{REFERENCES}

1. M. Tirrell, Rubber Chem. Technol., 57, 523(1984). See also many references cited.

2. L. Leger, H. Hervet, and F. Rondelez, Macromolecules, 14, 1732 (1981).

3. P. T. Callaghan and D. N. Pinder, Macromolecules, 17, 431 (1984).

4. J. A. Wesson, I. Noh, T. Kitano, and H. Yu, Macromolecules, 17, 782 (1984).

5. H. Kim, T. Chang, J. H. Yohanan, L. Wang, and H. Yu, Macromolecules, 19, 2737 (1986).

6. H. Deschamps and L. Leger, Macromolecules, 19, 2760 (1986).

7. C. R. Bartles, B. Christ, and W. W. Graessley, Macromolecules, 17, 2702 (1984).

8. (a) M. Antonietti, J. Coutandin, R. Grutter, and H. Sillescu, Macromolecules, 17, 798 (1984).

(b) M. Antonietti, J. Coutandin, and H. Sillescu,
Macromolecules, 19, 793 (1986).

9. (a) B. A. Smith, Macromolecules, 15, 469 (1982).

(b) B. A. Smith, E. T. Samulski, L.-P. Yu, and M. A. Winnik, Macromolecules, 18, 1901 (1985).

(c) B. A. Smith, S. J. Mumby, E. T. Samulski, and L.P. Yu, Macromolecules, 19, 470 (1986).

10. (a) P. F. Green, P. J. Mills, C. J. Palmstron, J. W. Mayer, and E. J. Kramer, Phys. Rev. Lett., 58, 2145 (1984).

(b) P. F. Green and E. J. Kramer, Macromolecules, 19, 1108 (1986).

11. G. Fleisher, Polym. Bull., 11, 75 (1984).

12. D. S. Pearson, G. VerStrate, E. von Meerwall, and F. G. Schilling, Macromolecules, 20, 1133 (1987).

13. Q. Trang-Cong, T. Chang, C. C. Han, and Y. Nishijima, Polymer, 27, 1705 (1986).

14. D. Axelrod, D. E. Koppel, J. Schlessinger, E. Elison, and W. W. Webb, Biophys. J., 16, 1055 (1976).

15. (a) B. A. Smith and H. M. McConnell, Proc. Natl. Acad. Sci. U.S.A., 75, 2759 (1978).

(b) B. A. Smith, W. R. Clark, and H. M. McConnell, Proc. Natl. Acad. Sci. U.S.A., 76, 5641 (1979).

16. M. Fragata, S. Ohnishi, K. Asada, and M. Takahashi, Biochemistry, 23, 4044 (1984).

17. S. Subramanian, M. Seul, and H. M. McConnell, Proc. Natl. Acad. Sci. U.S.A., 83, 1169 (1986).

18. K. W. Rhee, D. A. Gabriel, and C. S. Johnson, Jr., J. Phys. Chem., 88, 4010 (1984).

19. W. Urbach, H. Hervet, and F. Rondelez, J. Chem. Phys., 83, 1877 (1985).

20. J. Zhang, C. H. Wang, and D. Ehlich, Macromolecules, 19, 1390 (1986).

21. J. Zhang, C. H. Wang, and Z.-X. Chen, J. Chem. Phys., 85, 5359 (1986).

22. F. W. Wang, R. E. Lowry, and E.-S. Wu, Polymer, 26, 1654 (1986).

23. T. Inoue, N. Nemoto, T. Kojima, Y. Tsunashima, and M. Kurata, Nihon Reorogi Gakkaishi, 16, 72 (1988) (in Japanese).

24. T. Inoue, Ph. D. Thesis, Kyoto University (1988) (in Japanese).

25. N. Nemoto, Y. Tsunashima, and M. Kurata, Polym. J., 13, 827 (1981).

26. N. Nemoto, Y. Makita, Y. Tsunashima, and M. Kurata, Macromolecules, 17, 425 (1984).

27. N. Nemoto, S. Okada, T. Inoue, and M. Kurata, Macromolecules, 21, 1502, 1509 (1988). 